\title{
Performance Evaluation of the Ordinary Least Square (OLS) and Total Least Square (TLS) in Adjusting Field Data: An Empirical Study on a DGPS Data
}

\author{
M. S. Peprah ${ }^{1}$, I. O. Mensah ${ }^{1 *}$ \\ ${ }^{I}$ Deparment of Geomatic Engineering, University of Mines and Technology, Tarkwa, Ghana. \\ *Corresponding authors email: nanaobour20@yahoo.com; mspeprah@st.umat.edu.gh
}

DOI: $\underline{\text { http://dx.doi.org/10.4314/sajg.v6i1.5 }}$

\begin{abstract}
Survey measurements have become the traditional means of obtaining point positions by most surveyors over the centuries. The assertion that a post processed DGPS field data is precise, accurate and can be used to execute any engineering works due to its minimum human errors need to be reviewed; this is because the post processed field data still contains errors and needs to be adjust. Adjustments and computations is one of the main research field in mathematical and satellite geodesy to assess the magnitude of errors and to study their distributions whether they are within or not within the acceptable tolerance. In order to achieve the objective of this study, a DGPS field data was adjusted using the Ordinary Least Square (OLS) and Total Least Square (TLS) techniques. The OLS considers errors only in the observation matrix, and adjusts observations in order to make the sum of its residuals minimum. The TLS considers errors in both the observation matrix and the data matrix, thereby minimizing the errors in both matrices. The limited availability of information on OLS and TLS in adjusting DGPS field data and the uncertainty of which method is optimal, whether the OLS and TLS is the most appropriate technique has called for the need to undertake this study. This study aimed at comparing the working efficiency of the OLS and TLS, assessing their individual accuracy and selecting the most effective method in adjusting DGPS field data. Each model was assessed based on statistical indicators of mean horizontal error (MHE), mean bias error (MBE), mean absolute error $(M A E)$, root mean square error (RMSE), and standard deviation (SD). After applying the OLS and TLS methods independently for the same datasets, it was ascertained that, the OLS method was better in adjusting DGPS field data than the TLS with a MHE and SD of $+1.203079 m$ and $+1.663134 m$ as compared to TLS with MHE and SD of $+7.0985507 m$
\end{abstract}


and $+2.594045 m$ respectively. This study will therefore create opportunity for geospatial professionals to know the efficiency of OLS and TLS in solving some of the problems in mathematical and satellite geodesy.

Keywords: Differential Global Positioning, System, Total Least Square, Ordinary Least Square, Survey Adjustments, Horizontal Position Displacement.

\section{Introduction}

Survey measurements over the centuries have been the traditional means of measuring and portraying the earth's surface (Ghilani and Wolf, 2012). The measurement can be either direct or indirect (Ghilani, 2010). Survey measurements are one of the core mandate in all areas of geoscientific applications. The fundamental measured quantities in every survey measurements are distances, angles, and elevations. These forms the basis for coordinates determination of positions concerning a specific datum either horizontal or vertical (Annan et al., 2016a). From these coordinates positions, other distances and angles that were not obtained directly during field measurements may be computed indirectly. The errors that were present in the original direct observations may propagate by the computational process into the indirect values (Ghilani, 2010). Thus, the indirect measurements contain errors that are functions of the original errors (Ghilani, 2010). In adjusting the field data values, the traditional techniques that are normally used is the classical least squares techniques.

Classical least square techniques are the most widely used methods for adjusting field data of ground points. In the classical least square techniques, adjustment of the observation equations where only the observations are considered as stochastic (Acar et al., 2006). In some instances, the design matrix elements contain errors which are usually ignored in the classical least techniques and this ignorance remains as an uncertainty in the solution results (Acar et al., 2006).

Differential Global Positioning System (DGPS) is of a higher accuracy than the absolute observation due to the use of reference station where coordinates are known to ascertain accuracy. The techniques used in DGPS observations are static, fast static, stop and go, and real time kinematics. Among DGPS survey techniques, the static method is of 
a higher accuracy due to some techniques used in the data collection process. Data collected after field survey needs to be processed to obtain a desired result. DGPS data after post processing still contain errors (Ansah, 2016; Okwuashi, 2014). The errors remain in the data could be adjusted. Several adjustment methods exist such as ordinary least squares (Annan et al., 2016a; Okwuashi and Eyoh, 2012a), Total least squares (Acar et al., 2006; Annan et al., 2016b; Okwuashi and Eyoh, 2012b), robust estimation (Wieser and Brunner, 2001), least square collocation (LSC) (Moritz, 1972). Conversely, there is a common belief that data obtain from the GPS instrument are very precise and accurate due to less interference of humans in the collection process and can be used to execute any given task without further adjustments.

The measured field data like any other survey measurement contains errors and need to be adjusted. Usually, adjustment is done using classical approach such as ordinary least approach (Annan et al., 2016a; Okwuashi \& Eyoh, 2012b), but the ordinary least square which is based on regression analysis considers only the observations to be stochastic (Acar et al., 2006) and thus account for errors only in the observation vector. Conversely, there exist errors in both the design and observation matrices which ought to be modelled out (Annan et al., 2016a). OLS is the most commonly widely used adjustment method in geodesy. It has been used in many geodetic areas in the recent decades. Notable among them are approximation of the surfaces in engineering structures (Lenda, 2008), finding the relationship between global and Cartesian coordinates (Ziggah, 2012), predictions of local coordinates (Odutola et al., 2013), converting GPS data from global coordinate system to the National coordinate system (Dawod et al., 2011).

Another numerical method applicable for adjusting field data is the TLS. It is worth mentioning that several studies have been carried out by researchers with the TLS techniques. Notable among them are 3D datum transformation including the weighted scenarios (Amiri-Simkooei and Jazaeri, 2012), measuring data perturbation size (Markovsk et al., 2009), localization of robots (Yang, 1997), calibration of robots (Nievergelt, 1994). It was emphasized that OLS which was mostly used in early times gave less accuracies because it assumes errors only in the output variable, y-value, whereas TLS assumes errors in both the input and output variables (Effah, 2015). The total least squares (TLS) was invented to resolve the working efficiency of the OLS (Annan et al., 2016a). The TLS have the ability to adjust the errors in both the observation matrix and design matrix (Acar et al., 2006) in order to yield a better estimate. The limited availability of technical papers in 
geodesy on TLS have called for the need to undergone this study. Also, the optimal model in adjusting surveying networks whether the OLS and TLS is good enough is the objective of this present study. Researchers such as (Acar et al., 2006; Annan et al., 2016a, 2016b; Okwuashi and Eyoh, 2012; OKwuashi, 2014) have applied TLS to solve many scientific problems and they concluded the TLS working efficiency is encouraging. This present study adopted the OLS and TLS in adjusting DGPS network and to propose the models which is optimal for adjusting DGPS network.

Although extensive applications of OLS and TLS have been carried out, limited literature is available in geodesy technical papers on the applications of OLS and TLS, for adjusting DGPS field data especially in developing countries like Ghana where geodesy has not yet reached the advanced stage. In addition, there have been arguments on which method, whether the OLS or TLS model is the most effective based on their various ideologies. Thus, the TLS technique takes into account observational errors on both dependent and independent variables while OLS considers only the independent variable. Therefore, this present study aims at making a comparative study of both methods and selecting the most effective technique. The authors were motivated to embark this study because it is yet to be evaluated in Ghana. This study will also create the opportunity for geospatial professionals to arrive a conclusion on which technique is optimal in adjusting DGPS field after post processing.

\section{Study Area and Data Source}

The study area (Figure 1) is situated in the mining town of Tarkwa which is the administrative capital of the Tarkwa Nsuaem Municipal Assembly in the Western Region of Ghana. It is found in the Southwest of Ghana with geographical coordinates between longitudes $1^{\circ} 59^{\prime} 00^{\prime \prime} \mathrm{W}$ and latitude $5^{\circ} 18^{\prime} 00^{\prime \prime} \mathrm{N}$ and is $78 \mathrm{~m}$ above mean sea level. It is about $85 \mathrm{~km}$ from Takoradi, which is the regional capital, $233 \mathrm{~km}$ from Kumasi and about $317 \mathrm{~km}$ from Accra (Ziggah, 2012). The topography is generally described as remarkable series of ridges and valleys. The ridges are formed by the Banket and Tarkwa Phyllites whereas upper quartzite and Huni Sandstone are present in the valleys. Surface gradients of the ridges are generally very close to the Banket and Tarkwa Phyllites. Its environs generally lie within the mountain ranges covered by thick forest interjected by undulating terrain with few scarps. The study area has a South-western Equatorial climate with seasons influenced 
by the moist South-West Monsoon winds from the Atlantic Ocean and the North-East Trade Winds. The mean rainfall is approximately $1500 \mathrm{~mm}$ with peaks of more than $1700 \mathrm{~mm}$ in June and October. Between November and February, the rainfall pattern decreases to between $20 \mathrm{~mm}$ to $90 \mathrm{~mm}$ (Forson, 2006). The mean annual temperature is approximately $25^{\circ} \mathrm{C}$ with small daily temperature variations. Relative humidity varies from $61 \%$ in January to a maximum of $80 \%$ in August and September (Ziggah, 2012; Seidu, 2004).

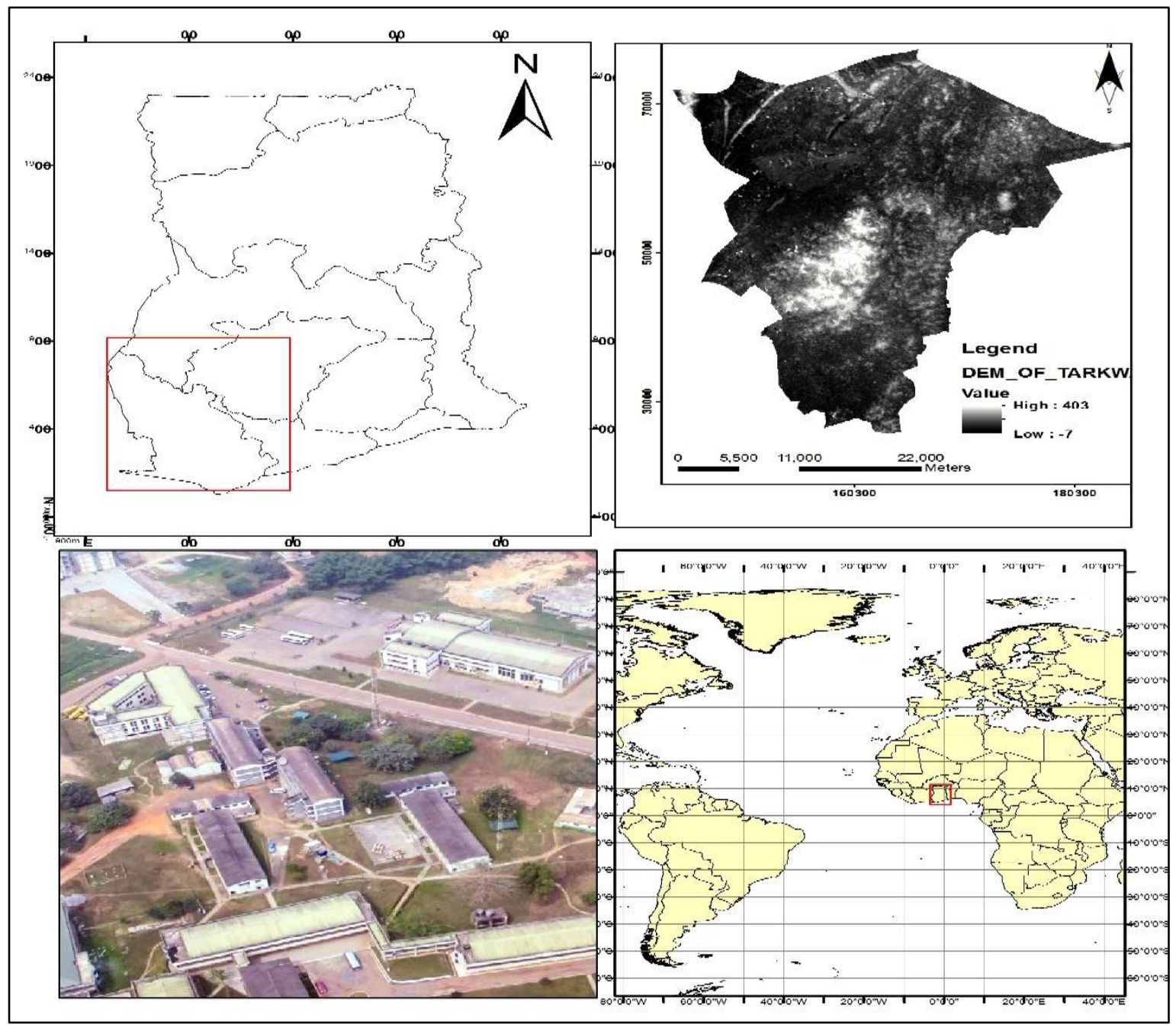

Figure 1. Study Area

In this study, a total of 12 DGPS data collected by field measurements in UMaT, Tarkwa, Ghana, situated in West Africa, were used in the OLS and TLS model formulation. It is well acknowledged that, one of the contributing factors affecting the estimation accuracy of models is related to the quality of datasets used in model-building (Dreiseitl and 
Ohno-Machado, 2002; Ismail et al., 2012). Therefore, to ensure that the obtained field data from the GPS receivers are reliable and accurate, several factors such as checking of overhead obstruction, obstruction, observation period, observation principles and techniques as suggested by many researchers (Yakubu and Kumi-Boateng, 2011; Ziggah et $a \mathrm{l} ., 2016)$ were performed on the field. In addition, all potential issues relating to GPS survey work were also considered.

\section{Methods}

\subsection{Ordinary Least Square (OLS) and Total Least Square (TLS)}

Least Square method is a statistical technique that is capable of determining the line of best fit of a model and seeks to find the minimum sum of the squares of residuals. This method is extensively used in regression analysis and estimation (Miller, 2006). Considering a system of equations in the form as denoted by Equation 1 to be solved by least squares:

$B X \approx L$

Where $B \in R^{m \times n}, X \in R^{n \times d}, L \in R^{m \times d}$, and $m \geq n$ (Annan et al., 2016a; Schaffrin, 2006). $B$ is the design matrix, $X$ is the matrix of the unknown parameters, and $L$ is the observation matrix.

The solution of the unknown parameters matrix X by OLS approach can be achieved as denoted by Equation 2:

$X=\left[B^{T} B\right]^{-1}\left[B^{T} L\right]$

The corresponding error vector $\mathrm{V}$ can be achieved by using Equation 3 as denoted by:

$V=B X-L$

On the other hand, solution of unknowns parameters $\hat{X}$ by TLS approach is obtained as denoted by Equation 4:

$L+V_{L}=\left[B+V_{B}\right] \widehat{X} \operatorname{rank}(B)=m<n$ 
Where $V_{L}$ is the error vector of observations and $V_{B}$ is the error matrix of the data matrix, the assumption that both have independently and identically distributed rows with zero mean and equal variance (Akyilmaz, 2007).

Golub and Van Loan, (1980), invented TLS to rectify the inefficiency associated with the OLS. Thus, accounting for perturbations in data matrix and observation matrix (Annan et al., 2016b). TLS is a mathematical algorithm that yields a unique solution in analytical form in terms of the Singular Value Decomposition (SVD) of the data matrix (Markovsky and Van Huffel, 2007). According to Golub and Van Loan, (1980) and Okwuashi and Eyoh, (2012a), the TLS algorithm is an iterative process which looks to minimize the errors in Equation 5 as denoted by:

$$
\min \|[B, L]-[\hat{B}, \hat{L}]\|_{F},[\hat{B}, \hat{L}] \in R^{n(m+1)}
$$

The optimization process goes on until a minimizing $[\hat{B}, \hat{L}]$ is obtained, any $\hat{X}$ that satisfies $\hat{B} \hat{X}=\hat{L}$ is the TLS solution (Annan et al., 2016a). In order to obtain the solution of $B \hat{X}=L$, we write the functional relation as denoted by Equation 6 :

$$
[B, L]\left[X^{T},-1\right]^{T} \approx 0
$$

The TLS problem can be solved using the Singular Value Decomposition (SVD) (Markovsky and Van Huffel, 2007; Ge and Wu, 2012). The SVD of the augmented matrix $[B, L]$ is required to determine whether or not it is rank deficient. Matrix $[B, L]$ can be represented by SVD as denoted by Equation 7 as:

$$
[B, L]=U S V^{T}
$$

Where $\mathrm{U}=$ real valued $\mathrm{m} \times \mathrm{n}$ orthonormal matrix, $\mathrm{UU}^{\mathrm{T}}=\mathrm{I}_{\mathrm{m}}, \mathrm{V}=$ real value $\mathrm{n} \mathrm{n}$ orthonormal matrix, $\mathrm{VV}^{\mathrm{T}}=\mathrm{I}_{\mathrm{n}}, \mathrm{S}=\mathrm{m} \mathrm{x} \mathrm{n}$ matrix with diagonals being singular values, offdiagonals are zeros. The rank of matrix $[B, L]$ is $\mathrm{m}+1$, and must be reduced to $\mathrm{m}$ using the Eckart-Young Mirsky theorem (Annan et al., 2016a). The TLS solution after the rank reduction is given by Equation 8 denoted as:

$$
\left[\hat{X}^{T},-1\right]=\frac{1}{V_{m+1, m+1}} V_{m+1}
$$


If $V_{m+1, m+1} \neq 0$, then $B X=L=-1 /\left(V_{m+1, m+1}\right) \cdot B\left[V_{1, m+1}, \cdots, V_{m, m+1}\right]^{T}$ belongs to the column space of $\hat{B}$, hence $\mathrm{X}$ solves the basic TLS problem (Okwuashi and Eyoh, 2012a). The corresponding TLS correction is achieved by using Equation 9 as denoted by:

$$
\lfloor\Delta \hat{B}, \Delta \hat{L}\rfloor=[B, L]-\lfloor\hat{B}-\hat{L}\rfloor
$$

\subsection{Models Performance Evaluation}

In order to determine the accuracies of the models used, the various statistical indicators were employed to determine the working efficiency of the models. Hence, to make an unprejudiced valuation of the models, statistical indicators such as Root Mean Square (RMSE), Mean Biased Error (MBE), and Mean Absolute Error (MAE), Horizontal Position error (HE), and Standard Deviation were used. Their individual mathematical languages are given by Equation 10 to Equation 14 respectively denoted by:

$$
R M S E=\sqrt{\sum \frac{E^{2}}{n}}
$$

where $\mathrm{n}$ is the number of observation points and $\mathrm{E}^{2}$ is the square of the error. The MBE was calculated using the formula below:

$$
M B E=\sum \frac{E}{N}
$$

where $\mathrm{E}$ is the error and $\mathrm{n}$ is the number of observation points. The MAE was calculated using the formula:

$$
M A E=\sum \frac{|E|}{N}
$$

where $|E|$ is the absolute error and $\mathrm{n}$ is the number of observation points.

$$
H E=\sqrt{\left(X_{2}-X_{1}\right)^{2}+\left(Y_{2}-Y_{1}\right)^{2}}
$$

Where $X_{2}$ and $Y_{2}$ are the existing coordinates, and $X_{1}$ and $Y_{1}$ are the observed coordinates.

$$
S D=\sqrt{\sum \frac{(x-\bar{x})^{2}}{n-1}}
$$


The Standard Deviation (SD) measures how closely the data are clustered around the mean. $n-1$ is the degree of freedom.

\section{Results and Discussions}

Table 1 shows the existing coordinates and the observed DGPS data. From Table 1, it was observed that, there is a difference between the existing data and the measured data. Table 2 and Table 3 shows the results obtained by the OLS and TLS models. The residual graphs for the OLS and TLS models are represented by Figure 2 and Figure 3. Figure 4 is the horizontal shift error graph obtained by the OLS and TLS models. From the graph, it was observed that the performance of the OLS model was encouraging as compared to the TLS.

Table 1. Existing Coordinates and DGPS field Data (Units in metres)

\begin{tabular}{|c|c|c|c|c|c|}
\hline ACTUAL & ACTUAL & DGPS & DGPS & \multicolumn{2}{|c|}{ RESIDUALS } \\
\hline $\mathbf{X}$ & $\mathbf{Y}$ & $\mathbf{X}$ & $\mathbf{Y}$ & $\boldsymbol{\Delta X}$ & $\boldsymbol{\Delta Y}$ \\
\hline 163244.2300 & 69656.2000 & 163244.2300 & 69656.2000 & 0.0000 & 0.0000 \\
\hline 163216.6680 & 69604.4470 & 163216.5376 & 69604.3286 & 0.1304 & 0.1184 \\
\hline 163363.5750 & 69380.3820 & 163363.1177 & 69380.1969 & 0.4573 & 0.1851 \\
\hline 163463.0150 & 69520.9960 & 163462.4604 & 69520.7708 & 0.5546 & 0.2252 \\
\hline 163505.7080 & 69583.2260 & 163505.2266 & 69582.9690 & 0.4814 & 0.2570 \\
\hline 163509.1960 & 69598.9950 & 163509.0747 & 69599.0941 & 0.1213 & -0.0991 \\
\hline 163517.3220 & 69634.4160 & 163515.8068 & 69635.0266 & 1.5152 & -0.6106 \\
\hline 163531.1310 & 69786.7810 & 163529.5562 & 69787.1048 & 1.5748 & -0.3238 \\
\hline 163497.2520 & 69817.9670 & 163494.9389 & 69818.4032 & 2.3131 & -0.4362 \\
\hline 163423.3670 & 69777.2060 & 163422.5800 & 69776.7900 & 0.7870 & 0.4160 \\
\hline 163394.6160 & 69753.3440 & 163392.7060 & 69753.3482 & 1.9100 & -0.0042 \\
\hline 163359.0890 & 69687.6660 & 163351.3071 & 69688.4810 & 7.7819 & -0.8150 \\
\hline
\end{tabular}

Table 2. Results obtained by the OLS models (Units in metres)

\begin{tabular}{|l|l|l|l|l|l|}
\hline ACTUAL & ACTUAL & \multicolumn{2}{|l|}{ ADJUSTED DGPS OLS } & \multicolumn{2}{l|}{ RESIDUALS } \\
\hline $\mathbf{X}$ & $\mathbf{Y}$ & $\mathbf{X}$ & $\mathbf{Y}$ & $\boldsymbol{\Delta} \mathbf{X}$ & $\Delta \mathbf{Y}$ \\
\hline 163244.2300 & 69656.2000 & 163245.8392 & 69656.6958 & -1.6092 & -0.4958 \\
\hline 163216.6680 & 69604.4470 & 163217.9890 & 69604.9549 & -1.3210 & -0.5079 \\
\hline 163363.5750 & 69380.3820 & 163363.7027 & 69380.4783 & -0.1277 & -0.0963 \\
\hline 163463.0150 & 69520.9960 & 163463.4563 & 69520.6158 & -0.4413 & 0.3802 \\
\hline 163505.7080 & 69583.2260 & 163506.4051 & 69582.6250 & -0.6971 & 0.6010 \\
\hline 163509.1960 & 69598.9950 & 163510.3055 & 69598.7258 & -1.1095 & 0.2692 \\
\hline
\end{tabular}




\begin{tabular}{|c|c|c|c|c|c|}
\hline 163517.3220 & 69634.4160 & 163517.1555 & 69634.6104 & 0.1664 & -0.1944 \\
\hline 163531.1310 & 69786.7810 & 163531.4144 & 69786.5363 & -0.2834 & 0.2447 \\
\hline 163497.2520 & 69817.9670 & 163496.9279 & 69817.9311 & 0.3241 & 0.0359 \\
\hline 163423.3670 & 69777.2060 & 163424.4771 & 69776.5937 & -1.1101 & 0.6123 \\
\hline 163394.6160 & 69753.3440 & 163394.5438 & 69753.2700 & 0.0722 & 0.0740 \\
\hline 163359.0890 & 69687.6660 & 163352.9523 & 69688.5891 & 6.1367 & -0.9231 \\
\hline
\end{tabular}

Table 3. Results obtained by the TLS model (Units in metres)

\begin{tabular}{|c|c|c|c|r|r|}
\hline & & \multicolumn{2}{|c|}{} & \multicolumn{2}{|c|}{} \\
\hline ACTUAL & ACTUAL & \multicolumn{1}{|c|}{ ADJUSTED DGPS TLS } & \multicolumn{2}{c|}{ RESIDUALS } \\
\hline $\mathbf{X}$ & $\mathbf{Y}$ & $\mathbf{X}$ & $\mathbf{Y}$ & \multicolumn{1}{c|}{$\boldsymbol{\Delta}$} & \multicolumn{1}{|c|}{$\boldsymbol{\Delta}$} \\
\hline 163244.2300 & 69656.2000 & 163247.7070 & 69664.4334 & -3.4770 & -8.2334 \\
\hline 163216.6680 & 69604.4470 & 163217.7791 & 69614.4181 & -1.1111 & -9.9711 \\
\hline 163363.5750 & 69380.3820 & 163352.0532 & 69385.4190 & 11.5218 & -5.0370 \\
\hline 163463.0150 & 69520.9960 & 163457.2129 & 69519.7871 & 5.8021 & 1.2089 \\
\hline 163505.7080 & 69583.2260 & 163502.5647 & 69579.2970 & 3.1433 & 3.9290 \\
\hline 163509.1960 & 69598.9950 & 163507.1551 & 69595.0755 & 2.0409 & 3.9195 \\
\hline 163517.3220 & 69634.4160 & 163515.5595 & 69630.3250 & 1.7625 & 4.0910 \\
\hline 163531.1310 & 69786.7810 & 163536.5335 & 69780.2261 & -5.4025 & 6.5549 \\
\hline 163497.2520 & 69817.9670 & 163503.7753 & 69812.8890 & -6.5233 & 5.0780 \\
\hline 163423.3670 & 69777.2060 & 163430.1214 & 69775.1919 & -6.7544 & 2.0141 \\
\hline 163394.6160 & 69753.3440 & 163399.4096 & 69753.4291 & -4.7936 & -0.0851 \\
\hline 163359.0890 & 69687.6660 & 163355.2823 & 69691.2106 & 3.8067 & -3.5446 \\
\hline
\end{tabular}

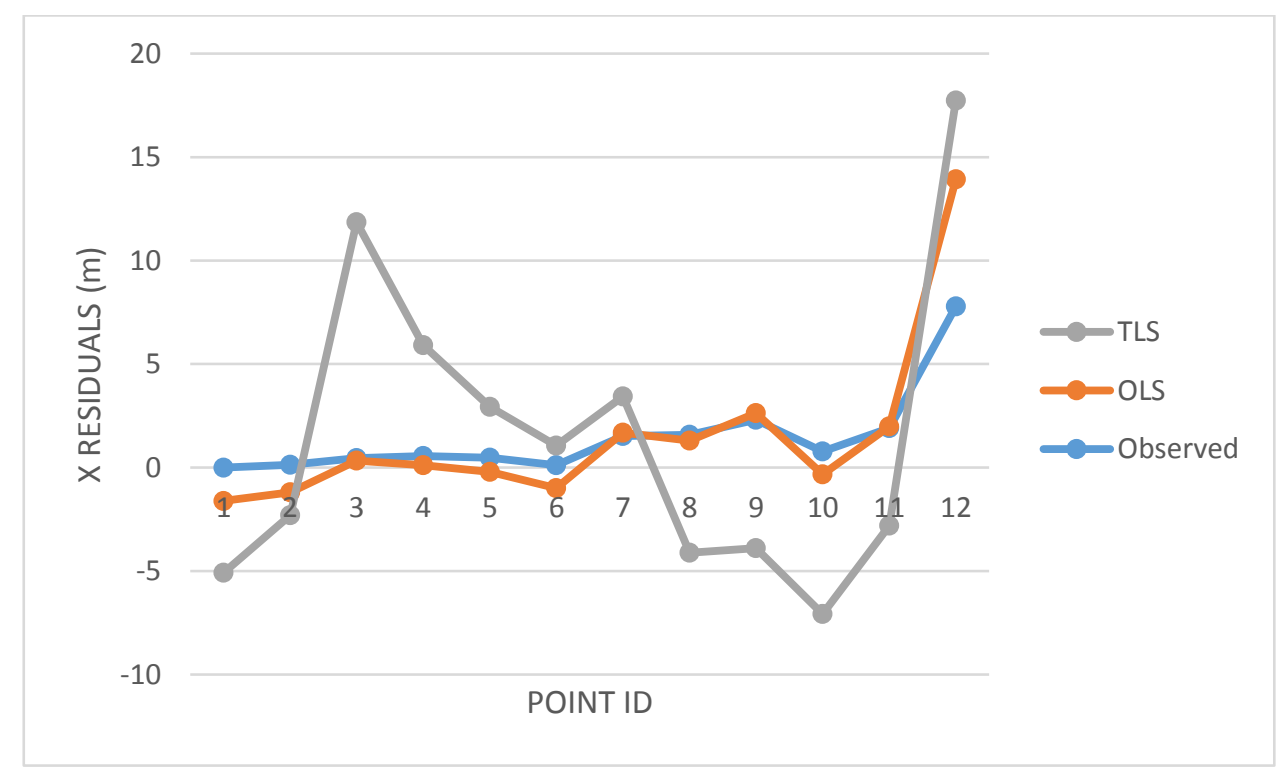

Figure 2 Residuals plot in the Eastings 
Figure 2 show the variations of residuals in eastings when the adjusted coordinates produced by the TLS and OLS methods respectively were subtracted from the actual existing coordinates. It was observed that the OLS method gave better estimates of the unknowns as compared to the TLS. This could possibly be that perturbation exists in the design matrix and the observation matrix formed from the coordinates obtained from the study area. Therefore, the OLS was preferred to that of the TLS for the study area.

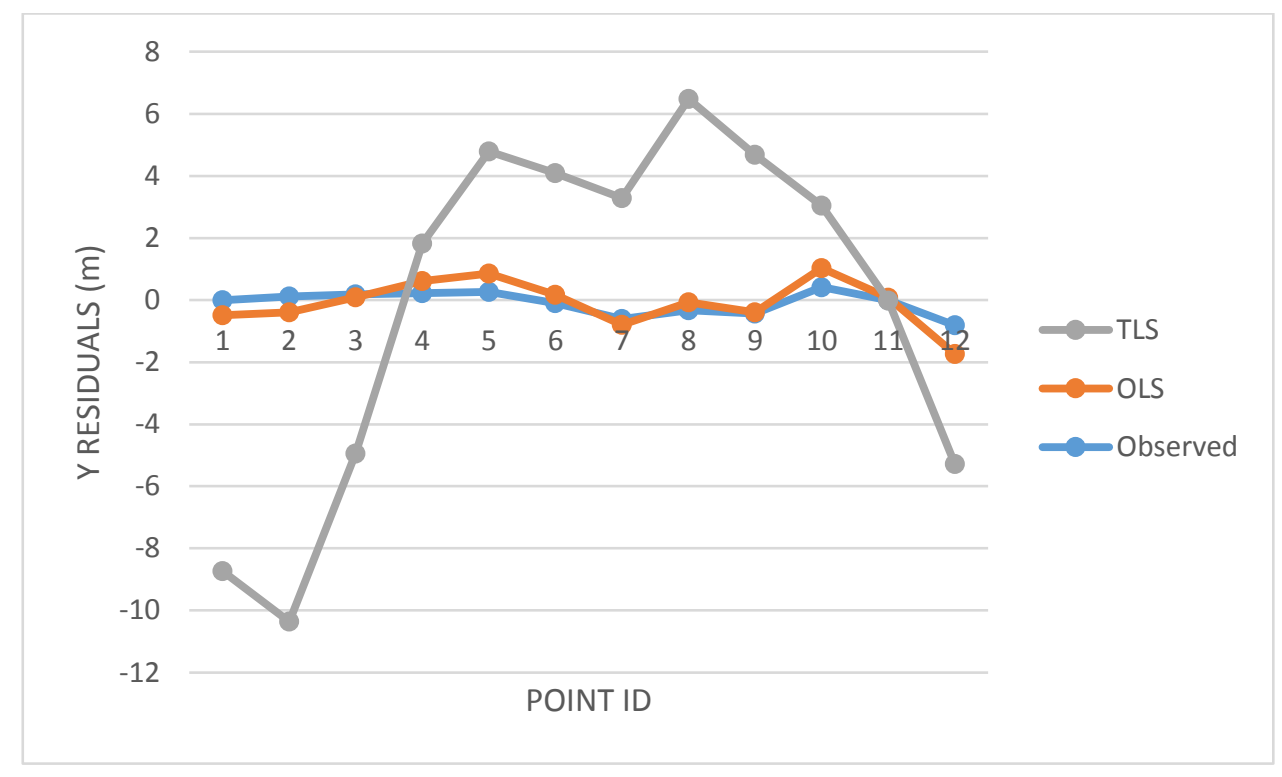

Figure 3 Residuals plot in the Northings

Similarly, Figure 3 show the variations of residuals in northings when the adjusted coordinates produced by the TLS and OLS methods respectively were subtracted from the actual existing coordinates. It was observed that the OLS method gave better estimates of the unknowns as compared to the TLS. This could possibly be that perturbation exists in the design matrix and the observation matrix formed from the coordinates obtained from the study area. Therefore, the OLS was preferred to that of the TLS for the study area.

The horizontal shifts of the positions for all the twelve points for both OLS and TLS methods were computed and the results is shown in Table 4. Figure 4 show the plot of the horizontal shift of the OLS and TLS models. 
Table 4 Horizontal Displacement of Points (Units in metres)

\begin{tabular}{|c|c|c|c|}
\hline POINT ID & OBSERVED & OLS & TLS \\
\hline 1 & 0.000000 & 1.683847 & 8.937472 \\
\hline 2 & 0.176133 & 1.415275 & 10.03282 \\
\hline 3 & 0.493341 & 0.159941 & 12.57471 \\
\hline 4 & 0.598578 & 0.582493 & 5.926703 \\
\hline 5 & 0.545706 & 0.920407 & 5.031637 \\
\hline 6 & 0.156635 & 1.141691 & 4.419022 \\
\hline 7 & 1.633604 & 0.255891 & 4.454513 \\
\hline 8 & 1.607744 & 0.374424 & 8.494335 \\
\hline 9 & 2.353870 & 0.326082 & 8.266772 \\
\hline 10 & 0.890183 & 1.267767 & 7.048299 \\
\hline 11 & 1.910005 & 0.103387 & 4.794335 \\
\hline 12 & 7.824461 & 6.205739 & 5.201457 \\
\hline
\end{tabular}

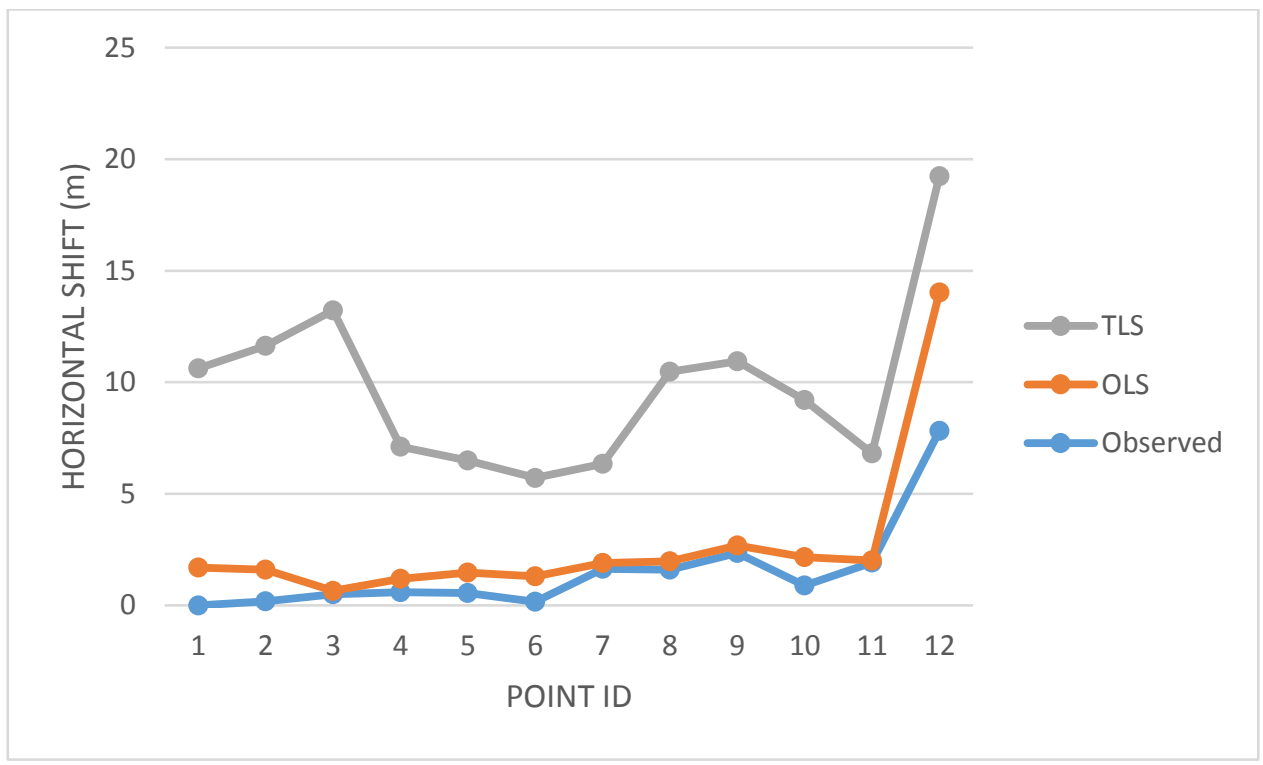

Figure 4 Horizontal Position error plot by the two models

Figure 4 represents the degree of horizontal positional accuracy in both OLS and TLS methods for the points obtained in the study area. A critical look at Tables 4 above revealed that the OLS method produced marginally better results than the TLS method. Therefore, the OLS method was preferred to that of the TLS for the study area. 
. In order to further access the statistical validity of the two models applied, the Mean Horizontal Position Error (MHE), Root Mean Square Error (RMSE), Mean Absolute Error (MAE), Mean Bias Error (MBE), and Standard Deviation (SD) were applied as the performance criteria index. The performance criteria index (PCI) values attained for computing the M, RMSE, MAE, MBE are shown in Table 5, Table 6 and Table 7 below.

Table 5. Statistical Model Validation of the observed data (Units in metres)

\begin{tabular}{|c|c|c|c|c|c|c|}
\hline PCI & M & MSE & MAE & MBE & RMSE & SD \\
\hline X & 1.468917 & 6.310989 & 1.468917 & 1.468917 & 2.512168 & 2.128578 \\
\hline Y & -0.090600 & 0.140009 & -0.090600 & -0.090600 & 0.374178 & 0.379187 \\
\hline HE & 1.515855 & 6.450998 & 1.515855 & 1.515855 & 2.539881 & 2.128554 \\
\hline
\end{tabular}

Table 6 Statistical Model Validation of the OLS model (Units in metres)

\begin{tabular}{|c|c|c|c|c|c|c|}
\hline PCI & M & MSE & MAE & MBE & RMSE & SD \\
\hline $\mathrm{X}$ & $8.333 \mathrm{E}-06$ & 3.781019 & $8.33 \mathrm{E}-06$ & $8.33 \mathrm{E}-06$ & 1.944484 & 2.030947 \\
\hline $\mathrm{Y}$ & $-1.66 \mathrm{E}-05$ & 0.201895 & $-1.66 \mathrm{E}-05$ & $-1.66 \mathrm{E}-05$ & 0.449327 & 0.469307 \\
\hline $\mathrm{HE}$ & 1.203079 & 3.982913 & 1.203079 & 1.203079 & 1.995724 & 1.663134 \\
\hline
\end{tabular}

Table 7. Statistical Model Validation of the TLS model (Units in metres)

\begin{tabular}{|c|c|c|c|c|c|c|}
\hline PCI & M & MSE & MAE & MBE & RMSE & SD \\
\hline X & 0.001283 & 29.31036 & 0.001283 & 0.001283 & 5.413904 & 5.654638 \\
\hline Y & -0.00632 & 27.24676 & -0.00632 & -0.00632 & 5.219843 & 5.451944 \\
\hline HE & 7.098507 & 56.55712 & 7.098507 & 7.098507 & 7.520447 & 2.594045 \\
\hline
\end{tabular}

From Table 5 to Table 7 above, it can be seen that the OLS gave a better result as compare to the TLS. The performance criteria indices of M, MSE, MAE, MBE, RMSE, and SD of OLS were lower than that of TLS, showing that OLS has a better performance for the Study area than TLS in this study. 


\section{Conclusions and Recommendation}

The conclusions made from this study is that, both the OLS and TLS methods have been utilized to adjust DGPS data. The performance of the two models developed were then compared based on statistical indicators of M, MSE, MAE, MBE, RMSE, and SD. The performance and efficiency of each adjustment techniques was assessed using an existing dataset that was not used to form the models. It was realized that, the OLS and TLS produced different results. This signifies that in terms of adjustments using the two models, there will be no identical results due to their working efficiencies and advantages over each other. However, it is recommended by the authors based on the results achieved in this study that, OLS is the proposed method for adjusting DGPS data for the study area, since the OLS method gave marginally better results than the TLS method. Therefore, the applicability of using least squares techniques to adjust DGPS data have been achieved in this study.

Based on the results and conclusions present in this study, it can be confidently and uncertainly say that, OLS method should be adapted in adjusting DGPS networks since it produced marginally better results than the TLS method. In addition, more research works should be conducted on other least squares techniques that was not adopted in this study such as partial least squares, generalized least squares, least squares collocation and many others and compared the outcomes to what was achieved in this study. This will further enhance the assessment of the least squares in selecting the most optimal among them for adjusting survey field data. This will study will therefore create opportunity for geospatial professional in geoscientific communities to realize the significance of least squares regressions models in solving some of the problems in mathematical and satellite geodesy.

\section{Acknowledgement}

The authors are very grateful and would like to thank the anonymous reviewers for their helpful comments to make this piece of knowledge a better material for academic purpose, and also to thank all geospatial professionals across the globe for their contributions and their impartation of knowledge related to this work and in all fields of geoscientific community. Special thanks go to the staff members of the Department of Geomatic Engineering, University of Mines and Technology, Tarkwa, Ghana, for providing us with the required knowledge and instruments used for the collection of data that was used to undertake this study. 


\section{References}

Acar, M., Ozuledemir, M. T., Akyilmaz, O., Celik, R. N., and Ayan, T. (2006), "Deformation analysis with Total Least Squares", Natural Hazards and Earth System Sciences, Vol. 6, pp. 663-669.

Akyilmaz, O. (2007), “Total Least Squares Solution of Coordinate Transformation”, Survey Review, Vol. 39, No. 303, pp. 68-80. http://doi.org/10.1179/003962607X165005

Amiri-Simkooel, A., and Jazaeri, S. (2012), "Weighted Total Least Squares Formulated by Standard Least Squares Theory", Journal of Geodetic Science, pp. 1-2.

Annan, R. F., Ziggah, Y. Y., Ayer, J., and Odutola, C. A. (2016a), “Accuracy Assessment of heights obtained from Total station and level instrument using Total Least Squares and Ordinary Least Squares Methods", Journal of Geomatics and Planning, Vol. 3, No. 2, pp. 87-92.

Annan, R. F., Ziggah, Y. Y., Ayer, J., Odutola, C. A. (2016b), “A Hybridized Centroid Technique for 3D Molodensky-Badekas Coordinate Transformation in the Ghana Reference Network using Total Least Squares Approach”, South African Journal of Geomatics, Vol. 5, No. 3, pp. 269-284.

Ansah, E. (2016), "DGPS Networks Adjustments using Least Squares Collocation", Unpublished BSc Project Work, University of Mines and Technology, Tarkwa, Ghana, $57 \mathrm{pp}$.

Dawod, G. M., Mirza, N. M., and Al-Ghamdi, A. K. (2011), "TS 8 Simple Precise Coordinate Transformations for Geomatics Applications in Makkah Metropolitan Area, Saudi-Arabia", Bridging the Gap Between Cultures FIG Working Week, 2010, Marrakech, Morocco, pp. 18-22.

Dreiseitl, S., and Ohno-Machado, L. (2002), "Logistic Regression and Artificial Neural Network Classification Models: A Methodology Review”, J Biomed Int, Vol. 35, No. 5-6, pp. 352-359.

Effah, S. F. (2015), “A Comparative Study of Ordinary Least Squares and Total Least Squares in Predicting 2D Cartesian Coordinates-A Case Study", Unpublished Thesis, University of Mines and Technology, Tarkwa, Ghana, 30 pp. 
Forson, K. I. (2006), "Design of distribution network for University of Mines and Technology", Unpublished BSc Project Report, University of Mines and Technology, Tarkwa, Ghana, 10pp.

Ge, X., and Wu, J. (2012), “A New Regularized Solution to Ill-Posed Problem in Coordinate Transformation", International Journal of Geosciences, Vol. 3, pp. 14-20.

Ghilani, D. C. (2010), “Adjustment Computations, Spatial Data Analysis”, Fifth Edition, Wiley \& Sons, INC. Hoboken, New Jersey, USA, 674 pp.

Ghilani, D. C., and Wolf, P. R. (2012), "Elementary Surveying, An Introduction to Geomatics. Thirteen Edition”, Pearson Education Inc., Upper Saddle River, New Jersey 07458, USA, 983 pp.

Golub, G. H., and Van Loan, C. F. (1980), “An analysis of the Total Least Squares problem”, SIAM Journal on Numerical Analysis, Vol. 17, No. 6, pp. 883-893.

Ismail, S., Shabri, A., and Samsudin, R. (2012), “A Hybrid Model of Self-Organizing Maps and Least Square Support Vector Machine for River Flow Forecasting”, Hydrol Earth Syst Sci, Vol. 16, pp. 4417-4433.

Lenda, G. (2008), “Application of Least Squares Method for Approximation the Surface Engineering Structures", Journal of Geomatic and Environmental Engineering, Vol. 2, No. 1, pp. 50-56.

Markovsky, I., and Van Huffel, S. (2007), "Overview of Total Least Square Methods", Signal Processing, Vol. 87, No. 10, pp. 2283-2302.

Makovsky, I., Sima, D. M., and Huffel, S. V. (2009), “Generalization of the Total Least Squares Problem, Advanced Reviewed Article, pp. 1-2.

Miller, S. J. (2006), "Methods of Least Squares", Statistics Theory, Cornell University, USA, Vol. 3, pp. 1-2.

Moritz, H. (1972), “Advanced least squares method Report”, No. 75, Dept. of Geodetic Science, OSU.

Nievergelt, Y. (1994), “Total Least Squares: State -of-the-Art Regression in Numerical Analysis", Society of Industrial and Applied Mathematics, Vol. 36, No. 2, pp. 258-264. 
Odutola, C. A., Beiping, W., and Ziggah, Y. Y. (2013), “Testing Simple Regression Model for Coordinate Transformation by Comparing its Predictive Result for Two Regions", Academic Research International, SAVAP International Publishers, Vol. 4, No. 6, pp. 540-549.

Okwuashi, O., and Eyoh, A. (2012a), “Application of total least squares to a linear surveying network", Journal of science and Arts, Vol. 4, No. 21, pp. 401-404.

Okwuashi, O., and Eyoh (2012b), "3D Coordinate transformation using total least squares", Academic Research International, Vol. 3, No. 1, pp. 399-405.

Okwuashi, O. (2014), “Adjustment Computation and Statistical Methods in Surveying”, A Manual in the Department of Geoinformatics \& Surveying, Faculty of Environmental Studies, University of Eyoh, Nigeria.

Schaffrin, B. (2006), “A note on Constrained Total Least Square estimation", Linear Algebra and Its Application, Vol. 417, pp. 245-258.

Seidu, M. (2004), "GIS as a Tool in Water Monitoring for Public Health and Safety Management", Unpublished BSc Report, University of Mines and Technology (UMaT), Tarkwa, Ghana, 6pp.

Yakubu, I., and Kumi-Boateng, B. (2011), "Control Position Fix using Single Frequency Global Positioning System Receiver Techniques - A Case Study", Res J Environ Earth Sci, Vol. 3, No. 1, pp. 32-37.

Yang, T. (1997), “Total Least Squares Filter for Robot Localization”, Digital Signal Processing Proceedings, 13th International Conference, Santorini, pp. 1-2.

Ziggah Y. Y. (2012), "Regression Models for 2-Dimensional Cartesian Coordinates Prediction: A Case Study at University of Mines and Technology (UMaT)", International Journal of Computer Science and Engineering Survey (ISCSES), Vol. 3, No. 6, 62pp.

Ziggah, Y. Y., Youjian, H., Yu, X., and Basommi, L. P. (2016b), "Capability of Artificial Neural Network for Forward Conversion of Geodetic Coordinates $(\varphi, \lambda, h)$ to Cartesian Coordinates (X,Y,Z),, Math Geosci, Vol. 48, pp. 687-721. 